\title{
Power Counting for Optimized Capacity in MBMS Enabled UTRAN
}

\author{
Andreas Panayides, Christophoros Christophorou, Josephine Antoniou, \\ Andreas Pitsillides, Vasos Vassiliou \\ University of Cyprus, Department of Computer Science \\ \{Panayides, Christophoros, Josephin,Andreas.Pitsillides,vasosv\}@cs.ucy.ac.cy
}

\begin{abstract}
MBMS bearer services can be provided in each cell by either Point-to-Point or Point-to-Multipoint transmission mode but for efficiency a decision has to be made between the two approaches. Initially, the fundamental criterion used to make this decision was the number of MBMS users in a cell (UE Counting). In this paper we extend our previous work, which proposed the power transmitted by the Base Station (BS) as the channel switching criterion (Power Counting). We evaluate our approach and illustrate its capability to dynamically adapt to continuously varying cell conditions by selecting the transmission mode in a more efficient way, preserving in this way the network's resources. At the same time we demonstrate the limitations of the conventional approach, in terms of radio resource efficiency. We show that by having the power used by the BS as the limiting factor we achieve optimized capacity during the transmission of MBMS bearer services in UTRAN.
\end{abstract}

\section{Introduction}

Multimedia Broadcast Multicast Service (MBMS) [1] is a new unidirectional (downlink only) bearer service introduced in UMTS (Universal Mobile Telecommunication System) Release 6 specifications [2], in which the same data is transmitted from a single source entity to multiple recipients allowing the network resources to be shared. According to [1], for radio resource efficiency, MBMS services can be provided in each cell by either Point-to-Multipoint ( $\mathrm{P}$ t-M) or by Point-to-Point (P-t-P) transmission, but for efficiency a decision has to be made between the two approaches. This decision is made by the RNC (Radio Network Controller). With the P-t-M transmission one Forward Access Channel (FACH) is established and shared by all the User Equipments (UEs) in the cell while with the P-t-P transmission one Dedicated Channel $(\mathrm{DCH})$ is established for each UE in the cell.
In P-t-M transmission the FACH channel covers the cell up to the cell's edge and transmits identical traffic to UEs receiving traffic in P-t-M mode. The traffic is transmitted in a multicast manner over this channel, i.e. it is not duplicated for each UE. The transmitted power for this channel is fixed and independent of the number of UEs, as there is no power control, but rather it is preset to ensure coverage up to the cell's edge. In contrast, in P-t-P transmission identical traffic is transmitted to each UE individually since each packet is duplicated and sent over each DCH established connection. DCH has the capability of power control, that is, the transmission power is adjusted accordingly to provide the required strength to reach the UE.

Based on the above, it is easy to conclude that P-t$\mathrm{M}$ transmission is efficient only when a large number of UEs belonging to the same multicast group is present in the cell, but is inefficient when used by a small number of UEs, especially when located close to the Base Station (BS). On the contrary, P-t-P transmission is efficient when used by a small number of UEs, but inefficient when used by a large number of UEs. However, the number of UEs and their location in a cell is time-dependant and can vary according to UE's mobility patterns. Also, idle UEs may join an ongoing MBMS session while others decide to leave. This greatly impacts on the BS's total transmitted power. Consequently, even if one transmission mode is used initially, a switch to the other transmission mode may be required to achieve efficient system operation. Given that transmission mode switching is essential, careful selection of the most efficient switching criterion must be employed.

The rest of the paper is organized as follows: Section 2 highlights the inefficiencies of the current approach and discusses its limitations. Section 3 describes the Power Counting algorithm and how it addresses these limitations. Section 4 provides a thorough performance evaluation of both schemes to illustrate optimized capacity gains when the Power Counting algorithm is employed. Finally, in section 5, conclusions and future work are presented. 


\section{Problem statement}

A popular approach for switching between P-t-P and P-t-M transmission modes, mainly due to its simplicity of implementation, has been the UE Counting approach (see [1]), i.e. the switching criterion is the number of UEs present in the cell and belonging to the same multicast group. This threshold is predetermined and it is often estimated by assuming the worst case scenario, i.e. all the users are located near the cell's edge. This is not a realistic approach because mobility and current location is not taken into account. As a result, UE Counting cannot address varying cell conditions whether these are translated as moving UEs or altering fading circumstances, both resulting in BS's transmitted power fluctuations. But even in situations when UEs are static, this doesn't necessarily mean that they are uniformly distributed, i.e. some UEs could be located near the cell's edge, while others near the BS.

Another important factor that needs to be examined is the possibility of the ping pong effect that could cause unacceptable signaling load in UTRAN, additional signaling load for physical channel reconfiguration via the air interface, and possibly even a further QoS degradation just because of the fact that a cell transmission mode switch is executing. The ping pong effect occurs when a switch between P-t-P and Pt-M takes place in short time intervals.

In general, finding an optimum threshold for triggering a transmission mode switch using the UE Counting algorithm is neither practical nor efficient; no mechanism is known that would allow tracking the state of the network at all times.

\section{Power Counting algorithm description}

With the above considerations in mind, we proposed a new algorithm in [3][4] and further enhanced and comprehensively evaluated it in an MBMS setting in this study. Similar ideas progressed to standardization in 3GPP TR 25.922 v7.1.0 [5] and were also investigated by other research groups [6].

The proposed algorithm addresses the drawbacks of UE Counting in order to deliver a capacity-efficient algorithm with acceptable QoS in terms of reliable MBMS content. Power Counting uses a different criterion from traditional UE Counting methods for switching: the BS's total transmitted power (hence Power Counting). During an MBMS session, the total transmitted power required to serve all participating UEs when DCH connections are established is continuously measured. The decisive factor, which triggers a mode switch, is which transmission mode involves the minimum transmission power, thus maximizing capacity.

A primary assumption considered in Power Counting is that when an MBMS session begins, the participating UEs are initialized in P-t-P mode. This is done because we assume that a group is more likely to have a small number of UEs participating at the beginning of an MBMS session rather than large one. After initialization, periodically the RNC decides if it is better to switch to P-t-M or remain in P-t-P mode.

The originally proposed algorithm [3][4] made a switching decision by taking into consideration the continuous average of power over the most recent interval of width equal to a "window" of $5 \mathrm{sec}$ (smoothed using a moving average filter). The moving average was used in order to minimize the probability of unnecessary switches due to a sudden increase of the power in DCH channels. It was also able to efficiently handle undesirable ping pong effects. The selection of a 5 seconds update period was based on the assumption (derived from empirical estimation) that UEs position cannot change significantly within this particular interval. However, the implementation of the algorithm presented here introduces adaptivity to address limitations of this approach, as described later: instead of considering the moving average over a fixed period, we compute the instantaneous power periodically (Period $T$ ) and address the possibility of a ping pong situation by introducing a parameter which defines the minimum inter-transition time between two consecutive switches. In a similar way, we gain control over the periods that a switch is triggered and successfully tackle the ping pong effect.

Period $T$ is an important parameter playing a key role in Power Counting algorithm's efficiency. Consider a pedestrian environment cell serving UEs moving with an average speed of $5 \mathrm{~km} / \mathrm{hr}$. Setting $T=5$ seconds might prove efficient in such a cell as the UEs cover a total distance of less than 7 meters in this period. However, the same period is significantly inefficient in a vehicular environment cell where UEs are moving with an average speed of $120 \mathrm{~km} / \mathrm{hr}$, thus covering 167 meters in 5 seconds, resulting in greater variations on the downlink power required. Thus, the selection of Period T should be depended on the cell's environment and be a function of the UEs' speed.

With Power Counting algorithm a switching decision occurs if the following conditions are met:

- If in P-t-P transmission mode and the estimated instantaneous DCHs total power (see below on how this is estimated) is $10 \%$ higher than the FACH power (the FACH power is known, as it is constant for a specific cell size) then a switch to P$\mathrm{t}-\mathrm{M}$ transmission mode is initiated. 
- If in P-t-M transmission mode and the estimated instantaneous DCHs total power is $10 \%$ less than the FACH power a switch to P-t-P transmission mode is initiated.

Besides being a second measure, which in conjunction with the inter-transition time parameter attempts to eliminate unnecessary switching (ping pong effect), this hysteresis zone introduced (equal to $10 \%$ of the FACH power) also aims to reduce traffic overheads due to a mode switching when the gain in power is not sufficient.

The DCH total transmitted power is the only metric that our algorithm needs to compute, since the power of the FACH channel is known and constant. When in $\mathrm{P}$-t-P mode it is easy to calculate the instantaneous total power of the DCH channels since the BS is aware of the downlink transmitted power used for each P-t-P link. Thus the power summation of all channels participating in P-t-P mode is periodically reported to the RNC, which then checks if the condition for switching to P-t-M transmission mode is met. On the other hand, when in P-t-M mode and given that the only established connection is that of the FACH channel, the question which rises is how we estimate the instantaneous downlink power of the DCHs connections since there is not any information about this in the BS. To address this, we use the same approach used by the downlink open loop power control [7]. With this approach, upon initiation of a recounting procedure by the RNC [1], the UEs will send received Ec/No CPICH signal strength measurement reports to the RNC. Upon receiving all the measurements reports the $\mathrm{RNC}$ estimates the total power that would be required for a P-t-P transmission mode by using the formula defined below:

$$
P_{T x}^{T o t}=\sum_{i=1}^{N}\left[\frac{R \times(E b / N o)_{D L}}{W} \times \frac{C P I C H_{-} T x}{C P I C H_{(E c / N o)_{-} i}}-\alpha \times P_{C a r}\right]
$$

Where:

- $N$ is the total number of the UEs receiving the MBMS Service in the P-t-M Cell

- $\boldsymbol{R}$ is the MBMS Service bit rate

- $(\boldsymbol{E b} / \mathbf{N o})_{D L}$ is the DL planned Eb/No value set for achieving a certain Bit Error Rate as to satisfy the required QoS

- $\boldsymbol{W}$ is the chip rate (3.84 Mcps)

- $\boldsymbol{C P I C H}_{(\mathrm{Ec} / \mathrm{No})_{-} i}$ is the measurement report received from UE

- CPICH_Tx is the initial power used for CPICH transmission

- $\boldsymbol{\alpha}$ is the orthogonality factor

- $\boldsymbol{P}_{\text {Car }}$ is the carrier power measured at the Node_B and reported to the RNC

\section{Performance Evaluation}

For the performance evaluation of the proposed scheme OPNET Modeller 11.0.A UMTS module [8] was used as a base for building the MBMS simulator [9]. The performance was evaluated by comparing the total downlink power (capacity) that becomes available when Power Counting is used instead of the existing UE Counting based on [1]. It worth mentioning that the UE Counting threshold is decided based on the worst case scenario i.e. all the users are assumed to be on the cell's edge. Usually and based on a series of scenarios that we had performed, this number cannot exceed 5 or 6 UEs. But in our scenarios, in order to better illustrate the benefits of our proposed Power Counting over the existing 3GPP UE Counting approach, different other thresholds are assumed. In order to illustrate the performance and the usefulness of the proposed approach three scenarios were simulated. Emphasis was given in mobility aspects and varying number of UEs participating in an MBMS session. Table 1 contains the details of the simulation setup.

Table 1. Cell simulation setup parameters used for all the illustrated scenarios

\begin{tabular}{|l|l|}
\hline \multicolumn{1}{|c|}{ Parameter } & \multicolumn{1}{c|}{ Value } \\
\hline Propagation Environment used & Outdoor Pedestrian Environment \\
\hline UEs Speed & $5 \mathrm{Km} / \mathrm{hour}$ \\
\hline Update Period & $5 \mathrm{sec}$ \\
\hline Shadow Fading Standard Deviation & $10 \mathrm{~dB}$ \\
\hline MBMS application (multicast traffic sent) & Streaming video (64 kbits/s) \\
\hline Transmission Time Interval (TTI) & $20 \mathrm{~ms}$ \\
\hline Cell radius & $1 \mathrm{Km}$ \\
\hline Sectorization & No \\
\hline Cell layout & Hexagonal \\
\hline Transport Channels & $\mathrm{DCH}$ and FACH \\
\hline FACH transmitted power & $2 \mathrm{~W}$ \\
\hline Max Node B transmitted power & $20 \mathrm{~W}$ \\
\hline & \\
\hline
\end{tabular}

Figure 1. 5 UEs moving from Cell 1 to Cell 2

Scenario 1 considers the case where a group of five UEs are moving from Cell 1 to Cell 2 (both initialized in P-t-P transmission mode) following the trajectory depicted in Figure 1. Three instances of this scenario have been simulated in order to illustrate the gain of the Power Counting over the UE Counting algorithm. In the first instance, the UEs (a number of twenty-nine UEs) are uniformly distributed in Cell 2, in the second instance the UEs are located near Cell's 2 edge, while in the third instance the UEs in Cell 2 are located near the BS. The results collected are illustrated in Figure 2 to Figure 4. The threshold for UE Counting switching, is assumed to be 34 UEs. Setting the threshold at this value will trigger a cell mode switch once the five UEs from Cell 1 enter Cell 2 since the criterion for a cell mode switch will be met.

For all the instances simulated, Power Counting performs better than the UE Counting algorithm, in terms of capacity (power) efficiency. With the first instance (Figure 2), where the UEs are uniformly 
distributed in the Target Cell (Cell 2), both algorithms execute the channel switching efficiently. However, the case with UE Counting is that the transmission mode remains in P-t-M mode since the number of the UEs meets and exceeds the condition to have a P-t-M transmission mode. This causes a significant amount of capacity waste since the UEs during their mobility towards the BS reduce the amount of power required considerably, making the use of a FACH redundant. Then again, the Power Counting algorithm, due to its ability to dynamically adapt to continuously varying cell conditions (UEs mobility towards the BS), selects the most efficient transmission mode, resulting in significant power savings, thus maximizing capacity.

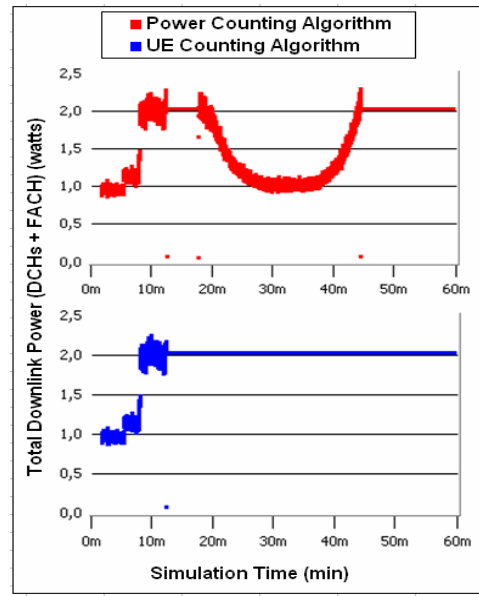

Figure 2. Total downlink power used (watts) - Power Counting vs UE Counting (Instance 1)

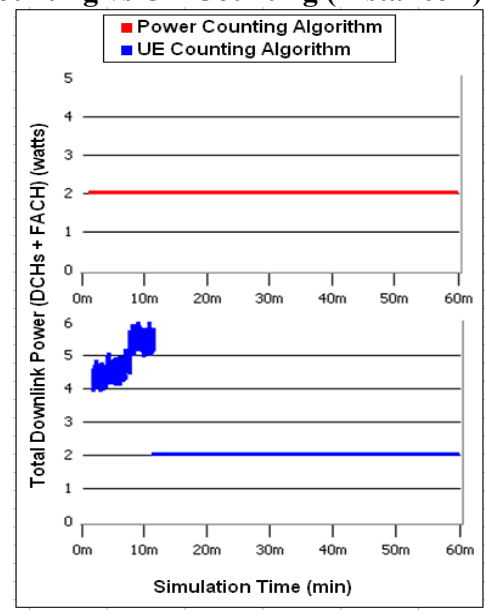

Figure 3. Total downlink power used (watts) - Power Counting vs UE Counting (Instance 2)

The capacity efficiency of the Power Counting is also depicted in Figure 3 (where the UEs are located near the cell's edge). As illustrated in this case, the initial power used in the cell varies between 4 and 4.9 watts. In this case the P-t-M mode is obviously the most efficient one (since the FACH allocates only 2 watts); the UE Counting algorithm keeps the transmission mode to P-t-P, until the number of the MBMS UEs in the cell is high enough in order to trigger the channel switching. The channel switching is finally executed at the $12^{\text {th }}$ minute of the simulation having reserved up to 6 watts of the total power of the BS. This capacity waste is avoided when the Power Counting algorithm is adopted, by establishing a P-t-M bearer in the cell from the beginning of the MBMS session. Then again, as shown in Figure 4 (where the UEs are located near BS), the Power Counting, in contrast with UE Counting, not only achieves efficient capacity, but also eliminates the need of channel switching, thus resulting in less signaling overhead.

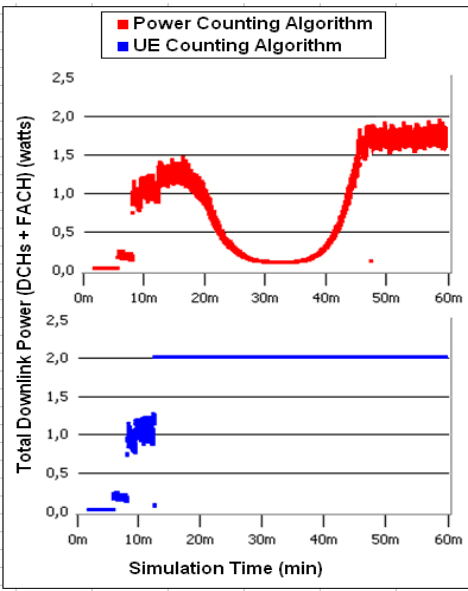

Figure 4. Total downlink power used (watts) - Power Counting vs UE Counting (Instance 3)

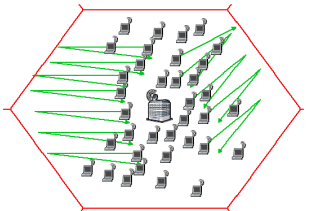

Figure 5. Eleven (11) UEs situated close to the BS are moving towards the cell edge and back

The second series of simulations includes a single cell serving 39 UEs. Eleven UEs move with a trajectory pointing to the cell edge and back (Figure 5). The goal is to depict the ability of the Power Counting algorithm to dynamically adapt to the varying conditions within a cell, likely to occur in real life deployed MBMS-enabled environments. Again, for evaluation purposes, simulations (incorporating parameters described in Table 1) were conducted both for UE Counting (two cases with threshold set to 50 and 30 respectively) and Power Counting algorithms. As discussed earlier in section 2, there exists no way of tracking the state of the network, thus selecting an optimum threshold at all times for the UE Counting algorithm. Therefore, the above thresholds are chosen aiming to depict both possible transmission modes (P$\mathrm{t}-\mathrm{P}$ and $\mathrm{P}-\mathrm{t}-\mathrm{M})$. As the UEs move towards the cell's edge and the distance from the BS increases, the power 
required for a DCH increases accordingly. The opposite happens once they start moving towards their original location closer to the BS. The results are displayed and analyzed in Figure 6 to Figure 8.

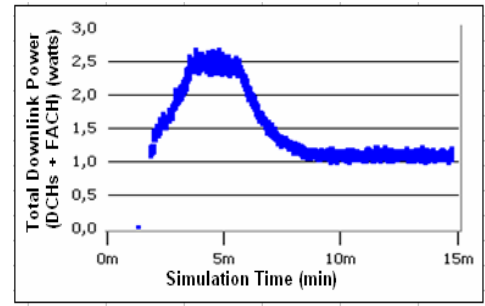

Figure 6. Total downlink power used by DCH and FACH channels (watts) versus time, UE Counting (50)

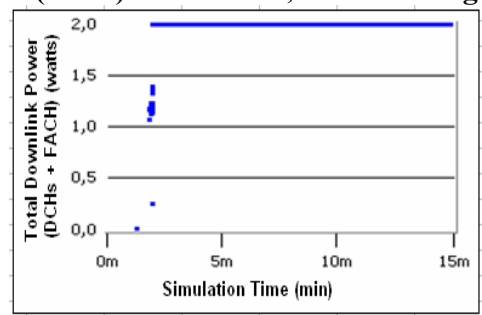

Figure 7. Total downlink power used by DCH and FACH channels (watts) versus time, UE Counting (30)

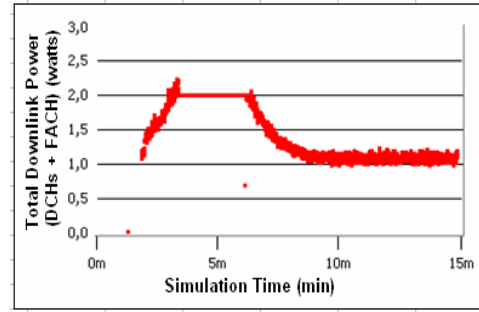

Figure 8. Total downlink power used by DCH and FACH channels (watts) versus time, Power Counting

Figure 6 records the total transmitted carrier power when the UE Counting algorithm with numeric threshold set to 50 is employed. Naturally, no cell mode switch is triggered, since the number of the participating UEs is lower than the provided threshold. Notice how the transmitted power increases as the UEs move away from the BS and decreases as the UEs follow the opposite direction. Using the same mode switching algorithm but with the numeric threshold set to 30, we observe (see Figure 7) that once the MBMS session begins, the cell switches to a P-t-M mode and remains in such for the duration of the simulation, regardless any mobility taking place within. That is because of the 39 established connections to the BS, which are greater than the numeric threshold set by the UE Counting algorithm for a cell mode switch. Figure 8 depicts the Power Counting algorithm's dynamic nature. Originally the cell is in a P-t-P mode. Once the UEs start moving away from the BS and the required power increases, continuous measurements (instantaneous power) allow effective switching to P-t$\mathrm{M}$ mode when the DCH channels total transmitted power surpasses that of the FACH channel by $10 \%$. In the reverse case (UEs returning closer to the BS), a P-t$\mathrm{M}$ to P-t-P switch is triggered, preserving in that way the network's resources. When compared to the UE Counting algorithm, up to a $6 \%$ gain in power is observed in the first case (no switching, P-t-P mode) and up to a $34 \%$ gain for the second case (switching to $\mathrm{P}-\mathrm{t}-\mathrm{M}$ for the whole simulation).

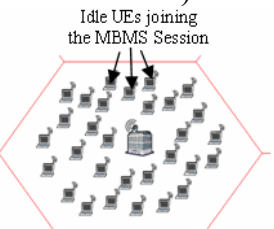

Figure 9. Idle UEs joining an ongoing MBMS session

In the third scenario (see Figure 9), we have one cell serving 34 UEs. Thirty one of them are receiving MBMS multicast traffic, while at the same time three are idle. The idle UEs join the MBMS session at the $450^{\text {th }}$ second of the simulation. Similarly to the above scenarios, simulations are carried out both for UE Counting (two cases with threshold set to 50 and 33 respectively) and UE Power Counting algorithms.

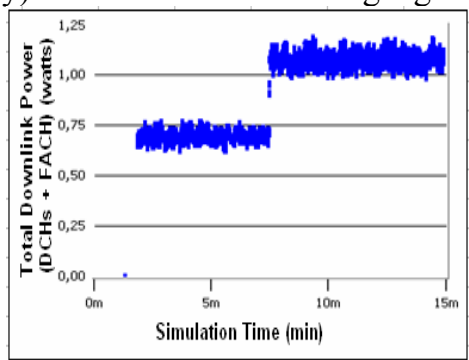

Figure 10. Total downlink power used by DCH and FACH channels (watts) versus time, UE Counting (50)

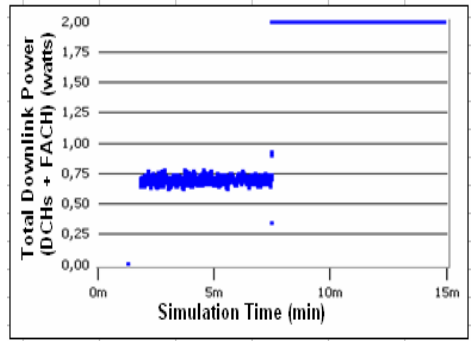

Figure 11. Total downlink power used by DCH and FACH channels (watts) versus time, UE Counting (33)

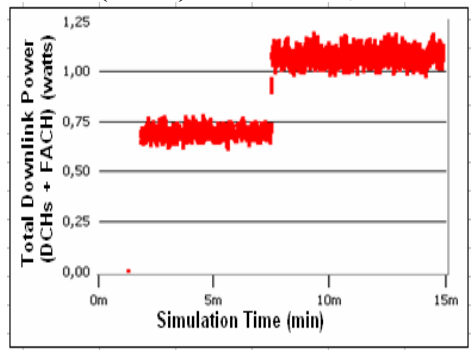

Figure 12. Total downlink power used by DCH and FACH channels (watts) versus time, Power Counting 
As expected, no switch is triggered for the UE Counting algorithm when the threshold is set to 50 (see Figure 10), since the threshold is never surpassed, even when the idle UEs join the session. However, when the numeric threshold is set lower to 33 UEs (Figure 11), once the idle UEs join the ongoing MBMS session, a P-t-P to P-t-M switch is triggered and the cell stays in P-t-M mode until the end of the simulation. On the other hand, when the Power Counting algorithm is employed, no switch is triggered (see Figure 12). That is because the UEs joining the ongoing MBMS session may cause the BS to transmit more power once they join the session (observe that they are located relatively away from the BS), however this increase in power does not justify a switch. Again, this scenario highlights the inability of the current UE Counting algorithm to address dynamic changes within the cell. Setting a higher threshold is not the answer either since a different scenario incorporating more distant UEs, may indeed require a mode transition which will not be triggered. Power Counting behaves better in the aforementioned case as well. For this particular scenario a $32 \%$ gain in terms of transmitted power is observed when compared to UE Counting algorithm (33) as well as a $44 \%$ reduction in peak transmitted power, however these gain values are scenario specific.

\section{Conclusions}

The Power Counting algorithm dynamically adjusts to varying cell conditions, since the total transmitted power for each mode is continuously monitored. In this manner, a capacity-efficient utilization of the network's resources is achieved. A channel mode switch is only triggered when a perceivable power gain is recorded and the possibility of undesired switches or ping pong effects is lessened. When compared to the UE Counting algorithm we observe that considerable power gain is attained in all simulated scenarios. Furthermore, the UE Counting approach is highly susceptible to the ping pong effect since no mechanism is proposed to prevent such incidents and a single UE, joining or leaving a session, may trigger consecutive cell mode switches. More importantly, adding a deadzone based on the UE count will make this approach even more inefficient.

The results presented above demonstrate substantial capacity gains when the Power Counting algorithm is employed. Further enhancements incorporate fine tuning of the periodicity the instantaneous power is measured (Period T) in different environments, as well as more comprehensive tests. Given that a power based approach could be undertaken during inter-cell mobility, namely "MBMS handover" [10], future work is also directed towards combining both approaches into a novel power preserving scheme optimizing system capacity in MBMS mode.

\section{Acknowledgement}

This work has been supported by IST-2005 27423 C-MOBILE and ICT-2007-216462 C-CAST projects. We also thank OPNET Technology Inc. for providing the software license to carry out the simulations of this research.

\section{References}

[1] 3GPP TS 25.346 v7.3.0, "Introduction of the Multimedia Broadcast Multicast Service (MBMS) in the Radio Access Network (RAN); Stage 2 (Release 7)", March 2007.

[2] 3GPP TS 23.101 V6.0.0, “General Universal Mobile Telecommunications System (UMTS) architecture (Release 6)", December 2004.

[3] N. Vlotomas, J. Antoniou, G. Hadjipollas, V. Vassiliou, A. Pitsillides. "Power Control for Efficient Multicasting in IP-based 3G and beyond mobile networks", 11th European Wireless 2005, Nicosia, Cyprus, April 10-13, 2005.

[4] B-BONE, Deliverable 3.2, "Report on Broadcast/Multicast RAN Resource Management Algorithms", August 2005.

[5] 3GPP TR 25.922 v7.1.0, "Radio Resource Management Strategies (Release 7)", Mar. 2007.

[6] A. Alexiou, C. Bouras, E. Rekkas, "A Power Control Scheme for Efficient Radio Bearer Selection in MBMS", IEEE International Symposium on a World of Wireless, Mobile and Multimedia Networks, Helsinki, Finland, June 2007.

[7] 3GPP TS 25.331 V7.5.0, "Radio Access Network; Radio Resource Control (RRC); Protocol Specification (Release 7), June 2007.

[8] OPNET University Program: http://www.opnet.com/services/ university/

[9] B-BONE MBMS System Level simulator, http://www.netrl.cs.ucy.ac.cy/

[10] A. Pitsillides, C. Christophorou, "MBMS Handover Control: A New Approach for Efficient Handover in MBMS Enabled 3G Cellular Networks", Computer Networks Journal (COMNET), Vol 51, Issue18, Pages: 4897-4918. 